\title{
Dam-break shock waves: a two-phase model for mature and immature debris flow
}

\author{
D. De Wrachien ${ }^{1} \&$ S. Mambretti ${ }^{2}$ \\ ${ }^{1}$ Department of Agricoltural Hydraulics, State University of Milan, Italy \\ ${ }^{2}$ DIIAR, Politecnico di Milano, Italy
}

\begin{abstract}
To predict flood and debris flow dynamics a numerical model, based on 1D De Saint Venant (SV) equations, modified for including erosion/deposition processes along the path, was developed. The McCormack-Jameson shock capturing scheme was employed for the solution of the equations, written in a conservative law form. This technique was applied to determine both the propagation and the profile of a two-phase debris flow resulting from the instantaneous and complete collapse of a storage dam. To validate the model, comparisons have been made between its predictions and laboratory tests concerning flows of water and homogeneous granular mixtures in a uniform geometry flume reproducing dam-break waves. Agreements between computational and experimental results are considered very satisfactory for mature (non-stratified) debris flows, which embrace most real cases. To better predict immature (stratified) flows, the model was improved in order to feature, in a more realistic way, the distribution of the particles of different size within the mixture. The level of maturity of the flow is assessed by an empirical, yet experimental based, criterion. The model, at this stage, should be able to predict the whole debris flow phenomenon, i.e. the triggering, mobilising and stopping processes of both mature and immature debris flows in different dam-break conditions. On the whole, the model proposed can easily be extended to channels with arbitrary cross sections for debris flow routing, as well as for solving problems of unsteady flow in open channels by incorporating the appropriate initial and boundary conditions. The model could also be improved to predict and assess the propagation and stoppage processes of debris and hyperconcentrated flows in mountainous catchments and river basins, triggered by extreme hydrological events, once validated on the basis of field data.
\end{abstract}

Keywords: debris flow, dam-break, two-phase modelling, mature and immature mixtures. 


\section{Introduction}

In this paper a $1 \mathrm{D}$ two-phase model for debris flow propagation is proposed. SV equations, modified for including erosion / deposition processes along the mixture path, are used for expressing conservation of mass and momentum for the two phases of the mixture. The scheme is validated for dam-break problems comparing numerical results with experimental data. Comparisons are made between both wave depths and front propagation velocities obtained respectively on the basis of laboratory tests and with predictions from the numerical model proposed by McCormack [18] and Jameson [13].

In order to analyze stratified (immature) flow - the solid/liquid mixture is present in the lower layer, while only water is present in the upper one - the model has been improved by taking into account mass and momentum conservation equations for each phase and layer. Momentum conservation equations describe energy exchanges between the two phases in the same layer and between layers, while mass conservation equations describe mass exchange layers $[16,17]$.

\section{Theoretical approach}

Debris flow resulting from flash floods such as a sudden collapse of a dam (dambreak) are often characterised by the formation of shock waves caused by many factors such as valley contractions, irregular bed slope and non-zero tailwater depth. It is commonly accepted that a mathematical description of these phenomena can be accomplished by means of 1D SV equations $[2,4,5]$.

Numerical treatments of such equations, generally, require schemes capable of preserving discontinuities, possibly without any special shift (shock-capturing schemes). Most numerical approaches have been developed in the last two or three decades, that include the use of finite differences, finite elements or discrete/distinct element methods $[1,19]$.

\subsection{Governing equations}

The 1D approach for unsteady debris flow triggered by dam-break is governed by the SV equations. This set of partial differential equations describes a system of hyperbolic conservation laws with source term (S) and can be written in compact vector form:

$$
\frac{\partial \mathbf{V}}{\partial t}+\frac{\partial \mathbf{F}}{\partial s}=\mathbf{S}
$$

where:

$$
\mathbf{V}=\left(\begin{array}{l}
A \\
Q
\end{array}\right) \quad \mathbf{F}=\left(\begin{array}{c}
Q \\
\frac{Q^{2}}{A}+g \cdot I_{1}
\end{array}\right) \quad \mathbf{S}=\left(\begin{array}{c}
0 \\
g \cdot A \cdot\left(i-S_{i}\right)+g \cdot I_{2}
\end{array}\right)
$$


with $A(s, t)$ : wetted cross-sectional area; $Q(s, t)$ : flow rate; $s$ : spatial coordinate; $t$ : temporal coordinate; $g$ : acceleration due to gravity; $i$ : bed slope; $S_{i}$ : bed resistance term or friction slope, that can be modelled using different rheological laws [19].

The pressure force integrals $I_{1}$ and $I_{2}$ are calculated in accordance with the geometrical properties of the channel. $I_{1}$ represents a hydrostatic pressure form term and $I_{2}$ represents the pressure forces due to the longitudinal width variation, expressed as:

$$
I_{1}=\int_{0}^{h}(H-\eta) \cdot \sigma(s, \eta) \cdot d \eta \quad I_{2}=\int_{0}^{h}(H-\eta) \cdot \frac{\partial \sigma}{\partial s} \cdot d \eta
$$

where $H$ : water depth; $\eta$ : integration variable indicating distance from the channel bottom; $\sigma(s, \eta)$ :channel width at distance $\eta$ from the channel bed, expressed as:

$$
\sigma(s, \eta)=\frac{\partial A(s, \eta)}{\partial \eta}
$$

To take into account erosion/deposition processes along the debris flow propagation path, which are directly related to both the variation of the mixture density and the temporal evolution of the channel bed, a mass conservation equation for the solid phase and a erosion/deposition model have been introduced in the SV approach. Defining the sediment discharge as:

$$
q(s, t)=E \cdot B
$$

with $E$ : erosion/deposition rate; $B$ : wetted bed width, the modified vector form of the SV equations can be expressed as follows:

$$
\frac{\partial \mathbf{V}}{\partial t}+\frac{\partial \mathbf{F}}{\partial s}=\mathbf{S}
$$

where:

$$
\mathbf{V}=\left(\begin{array}{c}
A \\
Q \\
c_{s} \cdot A
\end{array}\right) \quad \mathbf{F}=\left(\begin{array}{c}
Q \\
\frac{Q^{2}}{A}+g \cdot I_{1} \\
c_{s} \cdot Q
\end{array}\right) \quad \mathbf{S}=\left(\begin{array}{c}
q(s, t) \\
g \cdot A\left(i-S_{i}\right)+g \cdot I_{2} \\
E \cdot c_{*} \cdot B
\end{array}\right)
$$

with $c_{s}$ : volumetric solid concentration in the mixture; $c^{*}$ : bed volumetric solid concentration.

\subsection{Two phase mathematical model}

In the present work granular and liquid phases are considered. The model includes two mass and momentum balance equations for both the liquid and solid phases respectively. The interaction between phases is simulated according to the Wan and Wang [21] hypothesis. The system is completed with equations to estimate erosion / deposition rate derived from the Egashira and Ashida [7] relationship and by the assumption of the Mohr and Coulomb failure criterion for non cohesive materials. 


\subsubsection{Mass and momentum equations for the liquid phase}

Mass and momentum equations for water can be expressed in conservative form as:

$$
\begin{gathered}
\frac{\partial Q_{l}(s, t)}{\partial s}+\frac{\partial\left(c_{l} \cdot A(s, t)\right)}{\partial t}=0 \\
\frac{\partial Q_{l}}{\partial t}+\frac{\partial}{\partial s}\left(\beta \cdot \frac{Q_{l}{ }^{2}}{c_{l} \cdot A}\right)=g \cdot c_{l} \cdot A \cdot\left(i-J-\frac{\partial H}{\partial s}\right)-F
\end{gathered}
$$

with $Q_{l}(s, t)$ : flow discharge; $c_{l}$ : volumetric concentration of water in the mixture; $\beta$ : momentum correction coefficient that we will assume to take the value $\beta=1$ from now on; $J$ : slope of the energy line according to Chézy's formula; $i$ : bed slope; $F$ : friction force between the two phases.

According to Wan and Wang [21], the interaction of the phases at single granule level $f$ is given by:

$$
f=c_{D} \cdot \frac{\pi \cdot d_{50}^{2}}{4} \cdot \frac{\rho_{l} \cdot\left(v_{l}-v_{s}\right)}{2} \cdot\left|v_{l}-v_{s}\right|
$$

with $c_{D}$ : drag coefficient; $v_{l}$ : velocity of water; $v_{s}$ : velocity of the solid phase; $d_{50}$ : mean diameter of the coarse particle; $\rho_{l}$ : liquid density.

Assuming grains of spherical shape and defining the control volume of the mixture as:

$$
V_{c}=B \cdot H \cdot \cos \vartheta \cdot d s \approx B \cdot H \cdot d s
$$

with $\vartheta$ channel slope angle, which holds for low channel slopes, the whole friction force $F$ between the two phases for the control volume can be written as:

$$
F=\frac{3}{4} \cdot c_{D} \cdot \rho_{l} \cdot\left(v_{l}-v_{s}\right) \cdot\left|v_{l}-v_{s}\right| \cdot \frac{c_{s}}{d_{50}} \cdot H \cdot B \cdot d s
$$

\subsubsection{Mass and momentum equations for the solid phase}

Mass and momentum conservation equations for the solid phase of the mixture can be expressed as:

$$
\begin{gathered}
\frac{\partial\left(c_{s} \cdot A\right)}{\partial t}+\frac{\partial Q_{s}}{\partial s}=E \cdot c_{*} \cdot B \\
\frac{\partial Q_{s}}{\partial t}+\frac{\partial}{\partial s}\left(\beta \cdot \frac{Q_{s}^{2}}{c_{s} \cdot A}\right)=-g \cdot \frac{\rho_{s}-\rho_{l}}{\rho_{s}} \cdot c_{s} \cdot\left(1+i^{2}\right) \cdot \frac{\partial H}{\partial s} \cdot A+F+ \\
+g \cdot \frac{\rho_{s}-\rho_{l}}{\rho_{s}} \cdot c_{s} \cdot\left(i^{2}-1\right) \cdot \operatorname{tg} \delta \cdot A+g \cdot \frac{\rho_{s}-\rho_{l}}{\rho_{s}} \cdot c_{s} \cdot A \cdot i
\end{gathered}
$$

with $Q_{s}(s, t)$ : discharge of the solid rate; $\rho_{s}$ : solid phase density.

According to Ghilardi et at. [10] and to Egashira and Ashida [7], the bed volumetric solid concentration $c *$ was assumed to be constant and the erosion velocity rate $E$ a function of the mixture velocity $U$ :

$$
E=U \cdot k_{E} \cdot \operatorname{tg}\left(\vartheta_{f}-\vartheta_{e}\right)
$$

with $k_{E}$ : coefficient equal to 0.1 according to experimental data [7, 10-12]. 
Positive or negative values of $E$ correspond to granular material erosion or deposition, respectively.

$\vartheta_{f}$ and $\vartheta_{e}$ represent the energy line and the bed equilibrium angles, respectively, expressed as [6]:

$$
\begin{gathered}
\vartheta_{f}=\operatorname{arctg}\left[\frac{J}{\cos \vartheta}\right] \\
\vartheta_{e}=\operatorname{arctg}\left[\frac{c_{s} \cdot\left(\rho_{s}-\rho\right)}{c_{s} \cdot\left(\rho_{s}-\rho\right)+\rho} \cdot \operatorname{tg} \phi\right]
\end{gathered}
$$

where the debris flow density is defined as:

$$
\rho=\left(\rho_{s}-\rho_{l}\right) \cdot c_{s}+\rho_{l}
$$

and $\phi$ is the static internal friction angle. $U$ is defined as follows:

$$
U=c_{s} v_{s}+c_{l} v_{l}
$$

For $J$ the Takahashi [20] equation has been chosen, according to the dilatant fluid hypothesis developed by Bagnold [3]:

$$
J=S_{i}=\frac{U^{2}}{\left(\frac{2}{5 \cdot d_{50}} \cdot \frac{H}{\lambda}\right)^{2} \cdot \frac{1}{a_{b} \cdot \operatorname{sen} \delta} \cdot\left[c_{s}+\left(1-c_{s}\right) \cdot \frac{\rho_{l}}{\rho_{s}}\right] \cdot g \cdot R}
$$

with $S_{i}$ : friction term and $R$ : hydraulic radius given by:

$$
R=\frac{A}{P}
$$

where $P$ is the wetted perimeter.

The quantity $\lambda$ (linear concentration) depends on the granulometry of the solids in the form:

$$
\lambda=\frac{c_{s}{ }^{1 / 3}}{c_{m}^{1 / 3}-c_{s}^{1 / 3}}
$$

where $c_{m}$ : maximum packing volume fraction (for perfect spheres $c_{m}=0.74$ ); $a_{b}$ : empirical constant.

With regard to the momentum conservation equation (12) all its terms have been evaluated considering only the fraction of volume actually occupied by grains and ignoring the erosion/deposition velocity.

\section{Experimental results and model calibration}

To validate the model, comparisons have been made between its predictions and experimental results carried out in the Hydraulic Laboratory of the Politecnico di Milano. Numerical solutions of the SV equations are based on the well-known 
McCormack-Jameson predictor-corrector finite difference scheme ([13, 18]). The tests were performed with flows of water and homogeneous granular mixtures in a uniform geometry flume reproducing dam-break waves $([14,15])$. The experimental set-up consisted of a loading tank (dimensions $0.5 \mathrm{~m} \times 0.5 \mathrm{~m} \times$ $0.9 \mathrm{~m}$ ) with a downstream wall made of sluice gate, a pneumatic control device and a very short opening time $(0.3 s)$.

The mixture flowed in a $6 \mathrm{~m}$ long channel of square section $(0.5 \mathrm{~m} \times 0.5 \mathrm{~m})$ and adjustable slope. To enable camera recordings, one of the flume lateral walls contained glass windows.

Experimental tests were performed by changing the channel slope, the bottom roughness (smooth bottom made of galvanised plate or rough bottom covered with an homogeneous layer of gravel, with $d_{50}=0.005 \mathrm{~m}$ ), the solid material characteristics (vedril: $\rho=1168 \mathrm{~kg} / \mathrm{m}^{3}, d_{50}=0.003 \mathrm{~m}$; or gravel: $\left.\rho=2621 \mathrm{~kg} / \mathrm{m}^{3} \quad d_{50}=0.005 \mathrm{~m}\right)$ and the volumetric concentration of the mixture.

Recordings were made with a Sony Digital Handcam, model DCR-TRV32 E camera, which had an acquisition velocity of 25 frames per second, and were electronically elaborated.

To take into account different behaviours of the flow, the experimental data have been compared with the predictions of three rheological laws included in the one phase model (called "Water", "Fix Bagnold" and "Mobile Bagnold") and with those of the two phase model.

Comparisons show good agreement on the general shape that includes a steep front immediately followed by the maximum wave height and a decrease in flow depths down to an asymptotic value reached at the stoppage (figures 1 and 2).

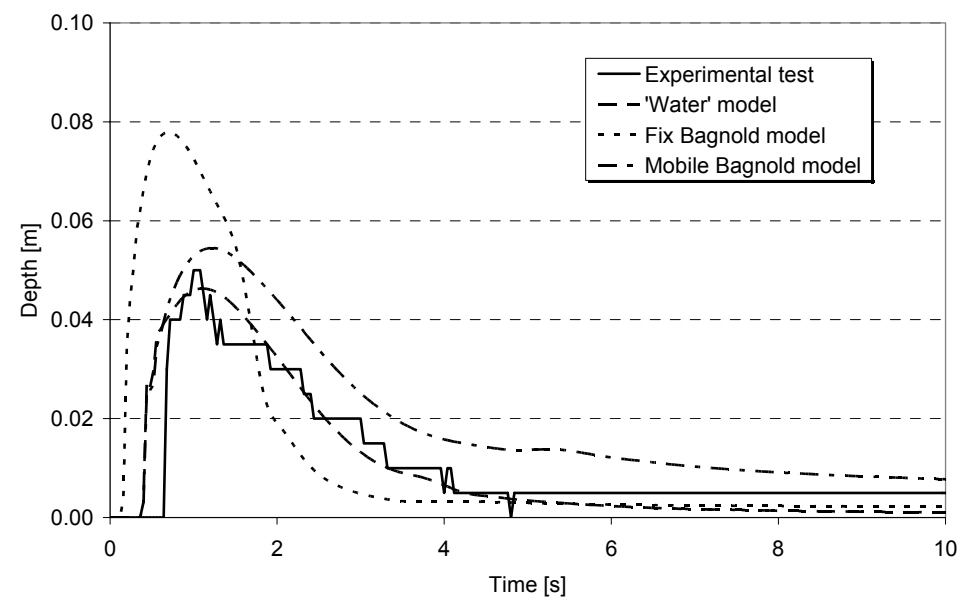

Figure 1: Debris flow wave in some characteristic sections of the experimental channel. Comparison between mathematical model and experimental results. Water-gravel, abs 200 , conc. $40 \%$, slope $15^{\circ}$, smooth bottom. 


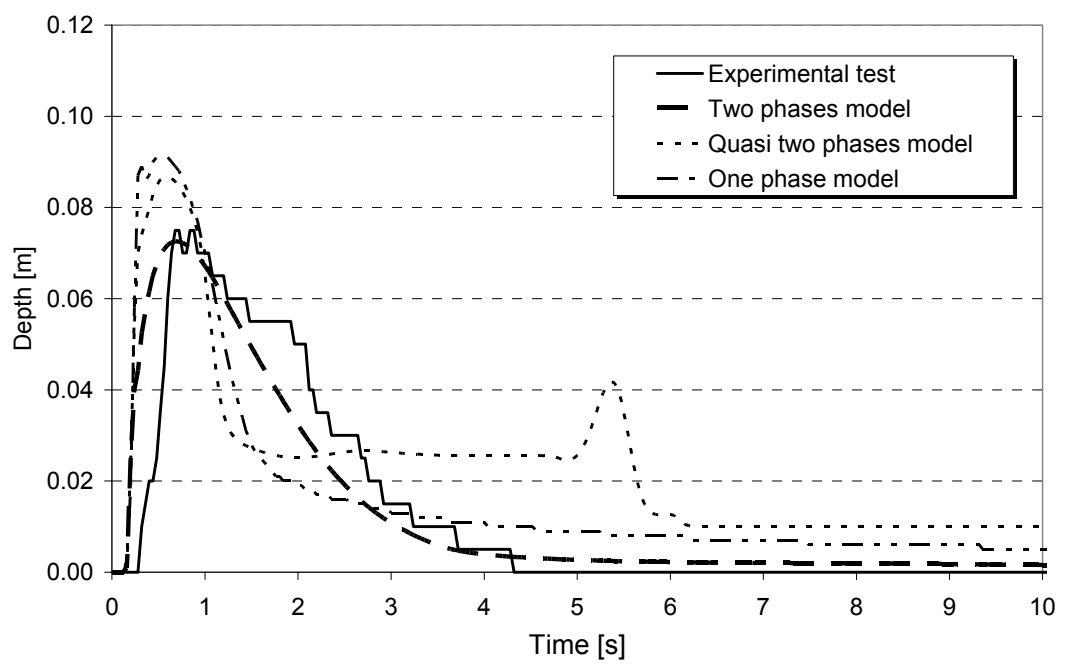

Figure 2: Debris flow wave in some characteristic sections of the experimental channel. Comparison between mathematical model and experimental results. Water-gravel, abs 140 , conc. $40 \%$, slope $20^{\circ}$, smooth bottom.

\section{Further development of the model}

One of the main features of this paper is to present a two-phase mathematical model, based on the SV equations, suitable to describe the propagation and the profile of debris flow resulting from flash floods such as a sudden collapse of a dam (dam-break). Such an approach has been validated on the ground of laboratory tests, for mature (non-stratified) debris flow. This evidently puts the bases for future research activity and the challenge is to make the tool able to reach, with regard to stratified (immature) flows, the same reliability up to now achieved for the mature ones.

\subsection{Stratified (immature) flows}

Debris flows are categorized as stratified or immature whenever the solid/liquid mixture is present in the lower layer, while only the water is present in the upper one (figure 3).

Assuming $h_{m x}$ and $h_{c w}$ as the depths of the mixture and of the clear water respectively, the total depth of the debris flow $h_{d f}$ is equal to:

$$
h_{d f}=h_{m x}+h_{c w}
$$

while the maturity degree is assessed as the ratio:

$$
d_{m}=\frac{h_{m x}}{h_{d f}}
$$


Larcan et al [15] has suggested - on the basis of laboratory experiments - to distinguish mature and immature debris flow by means of a criterion based on mixture velocity and concentration (figure 4).

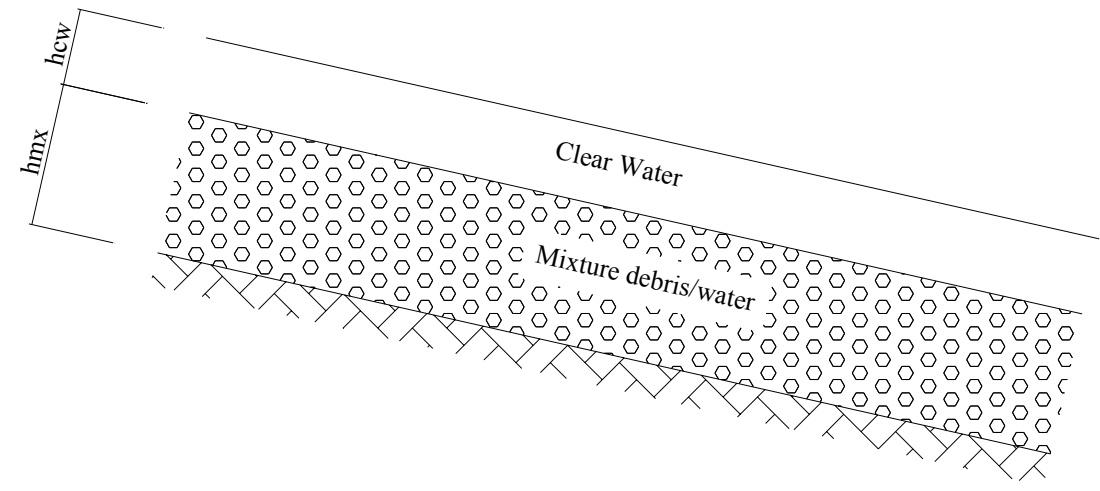

Figure 3: $\quad$ Scheme of the immature (stratified) debris flow.

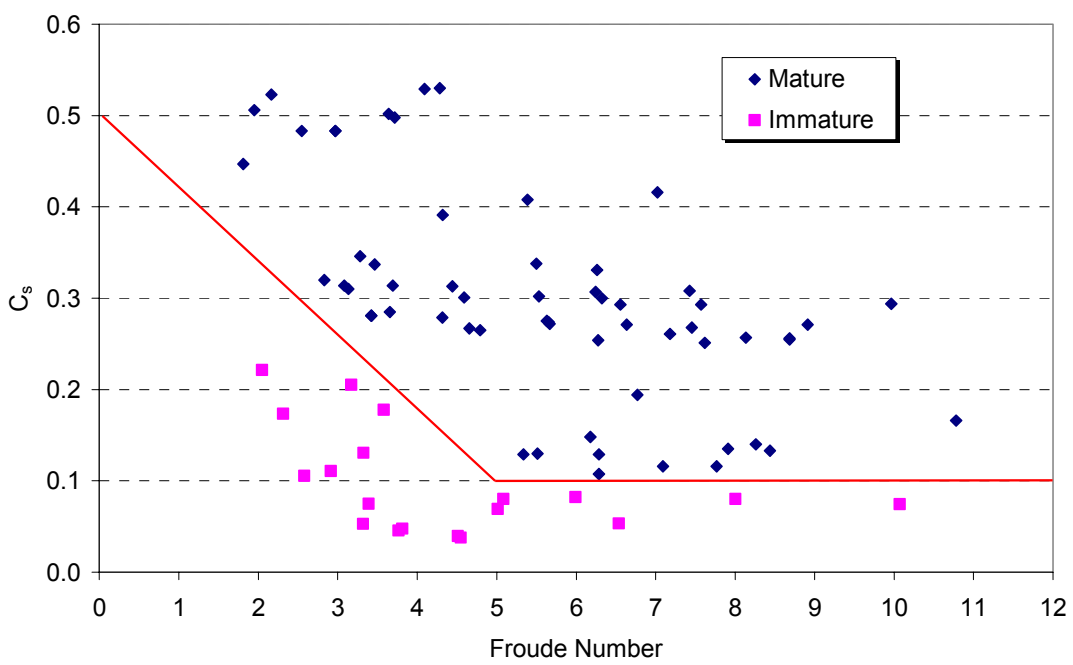

Figure 4: Characteristics of mature and immature debris flows.

The figure underlines the effectiveness of the above mentioned criterion and depicts a boundary between mature and immature debris flow. The boundary can be expressed by:

$$
C_{s \text { boundary }}=\left\{\begin{array}{lll}
0.5-0.08 \cdot F r & \text { when } & F r<5 \\
0.1 & \text { when } & F r \geq 5
\end{array}\right.
$$


with $F r$ : Froude number, while the maturity degree can be assessed as:

$$
d_{m}=\frac{C_{s \text { effective }}}{C_{s \text { boundary }}}
$$

The experimental tests showed that in the first phase the flow is stratified; then, usually, it becomes mature, because the velocities and the concentrations are quite high. Finally, the tail of the wave is characterised by low velocities, due to the fact that the solid phase tends to deposit, and thus the flow becomes again stratified.

\subsection{Mass and momentum equations for the liquid phase-higher layer (cw)}

Mass and momentum equations for clear water can be expressed in conservative form as:

$$
\begin{gathered}
\frac{\partial Q_{c w}(s, t)}{\partial s}+\frac{\partial A_{c w}(s, t)}{\partial t}=0 \\
\frac{\partial Q_{c w}}{\partial t}+\frac{\partial}{\partial s}\left(\beta \cdot \frac{Q_{c w}{ }^{2}}{A_{c w}}\right)=g \cdot A_{c w} \cdot\left(i-J_{c w}-\frac{\partial H_{c w}}{\partial s}-J_{\text {two layers }}\right)
\end{gathered}
$$

The resistance term $J_{c w}$ can be assessed on the basis of bank shear stress, while the slope of the energy line, $J_{\text {two layers }}$, due to the lower layer, according to Chézy's formula, is expressed as:

$$
J_{\text {two layers }}=\frac{n^{2} \cdot\left(V_{c w}-V_{m x}\right)^{2}}{R^{4 / 3}}
$$

being $n$ the Manning's number and $V_{m x}$ the velocity of the lower layer. The drag force $T_{\text {two layers }}$ between the higher layer and the lower one, can be expressed as:

$$
T_{\text {two layers }}=g \cdot A_{c w} \cdot J_{\text {two layers }}
$$

\subsection{Mass and momentum equations for the liquid phase-lower layer ( $\mathrm{mx})$}

In the same ways as (6) and (7), these equations can be expressed as:

$$
\begin{gathered}
\frac{\partial Q_{l, m x}(s, t)}{\partial s}+\frac{\partial\left(c_{l, m x} \cdot A_{m x}(s, t)\right)}{\partial t}=0 \\
\frac{\partial Q_{l, m x}}{\partial t}+\frac{\partial}{\partial s}\left(\beta \cdot \frac{Q_{l, m x}{ }^{2}}{c_{l, m x} \cdot A_{m x}}\right)=g \cdot c_{l, m x} \cdot A_{m x} \cdot\left(i-J_{m x}-\frac{\partial H_{m x}}{\partial s}\right)+ \\
-F+c_{l, m x} \cdot T_{\text {two layers }}
\end{gathered}
$$

$T_{\text {two layers }}$ is opposite in sign with respect to (28) due to the fact that the higher layer, with greater velocities, exerts a drag force to the mixture.

\subsection{Mass and momentum equations for the solid phase-lower layer}

Likewise (11) and (12), mass and momentum equations can be expressed as:

$$
\frac{\partial\left(c_{s, m x} \cdot A_{m x}\right)}{\partial t}+\frac{\partial Q_{s, m x}}{\partial s}=E \cdot c_{*} \cdot B
$$


192 Monitoring, Simulation, Prevention and Remediation of Dense Debris Flows II

$$
\begin{aligned}
& \frac{\partial Q_{s, m x}}{\partial t}+\frac{\partial}{\partial s}\left(\beta \cdot \frac{Q_{s, m x}^{2}}{c_{s, m x} \cdot A_{m x}}\right)=-g \cdot \frac{\rho_{s}-\rho_{l}}{\rho_{s}} \cdot c_{s, m x} \cdot\left(1+i^{2}\right) . \\
& . \frac{\partial H_{m x}}{\partial s} \cdot A_{m x}+F+g \cdot \frac{\rho_{s}-\rho_{l}}{\rho_{s}} \cdot c_{s, m x} \cdot\left(i^{2}-1\right) \cdot \operatorname{tg} \delta \cdot A_{m x}+ \\
& +g \cdot \frac{\rho_{s}-\rho_{l}}{\rho_{s}} \cdot c_{s, m x} \cdot A_{m x} \cdot i+c_{s, m x} \cdot T_{\text {two layers }}
\end{aligned}
$$

\subsection{Numerical model}

The SV equations for 1D two-phase unsteady debris flow can be expressed in compact vector form as follows:

$$
\frac{\partial \mathbf{V}}{\partial t}+\frac{\partial \mathbf{F}^{\prime}}{\partial s}+C \cdot \frac{\partial \mathbf{F}^{\prime \prime}}{\partial s}=\mathbf{S}
$$

where, for a rectangular section channel and for a completely mixed fluid:

$$
\begin{aligned}
& \mathbf{V}=\left(\begin{array}{c}
c_{l} \cdot A \\
c_{s} \cdot A \\
Q_{l} \\
Q_{s}
\end{array}\right) \quad \mathbf{F}^{\prime}=\left(\begin{array}{c}
Q_{l} \\
Q_{s} \\
\frac{Q_{l}{ }^{2}}{c_{l} \cdot A} \\
\frac{Q_{s}{ }^{2}}{c_{s} \cdot A}
\end{array}\right) \quad \mathbf{F}^{\prime \prime}=\left(\begin{array}{c}
0 \\
0 \\
\frac{1}{2} \cdot g \cdot \frac{A^{2}}{B} \\
\frac{1}{2} \cdot g \cdot \frac{\rho_{s}-\rho_{l}}{\rho_{s}} \cdot\left(1+i^{2}\right) \cdot \frac{A^{2}}{B}
\end{array}\right) \\
& \mathbf{S}=\left(\begin{array}{l}
0 \\
E \cdot c_{*} \cdot B \\
g \cdot c_{l} \cdot A \cdot(i-J)-\frac{3}{4} \cdot c_{D} \cdot\left(v_{l}-v_{s}\right)^{2} \cdot \frac{c_{s} \cdot A}{d_{50}} \\
g \cdot c_{s} \cdot A \cdot \frac{\rho_{s}-\rho_{l}}{\rho_{s}} \cdot\left[\left(i^{2}-1\right) \cdot \operatorname{tg} \delta+i\right]+ \\
+\frac{3}{4} \cdot c_{D} \cdot \frac{\rho_{l}}{\rho_{s}} \cdot\left(v_{l}-v_{s}\right)^{2} \cdot \frac{c_{s} \cdot A}{d_{50}}
\end{array}\right)
\end{aligned}
$$

and for a stratified (immature) flow:

$$
\mathbf{V}=\left(\begin{array}{c}
A_{c w} \\
c_{l, m x} \cdot A_{m x} \\
c_{s, m x} \cdot A_{m x} \\
Q_{c w} \\
Q_{l, m x} \\
Q_{s, m x}
\end{array}\right) \quad \mathbf{F}^{\prime}=\left(\begin{array}{c}
Q_{c w} \\
Q_{l, m x} \\
Q_{s, m x} \\
\frac{Q_{c w}^{2}}{A_{c w}} \\
\frac{Q_{l, m x}{ }^{2}}{c_{l, m x} \cdot A_{m x}} \\
\frac{Q_{s, m x}{ }^{2}}{c_{s, m x} \cdot A_{m x}}
\end{array}\right)
$$




$$
\begin{aligned}
& \mathbf{F}^{\prime \prime}=\left(\begin{array}{c}
0 \\
0 \\
0 \\
\frac{1}{2} \cdot g \cdot \frac{A_{c w}^{2}}{B} \\
\frac{1}{2} \cdot g \cdot \frac{A_{m x}^{2}}{B} \\
\frac{1}{2} \cdot g \cdot \frac{\rho_{s}-\rho_{l}}{\rho_{s}} \cdot\left(1+i^{2}\right) \cdot \frac{A_{m x}^{2}}{B}
\end{array}\right) \\
& \left(\begin{array}{c}
0 \\
0 \\
E \cdot c_{*} \cdot B \\
g \cdot A_{c w} \cdot\left(i-J_{c w}\right)-\frac{n^{2} \cdot\left(V_{c w}-V_{m x}\right)^{2}}{4 / 3} \\
R_{c w}^{4 / 3}
\end{array}\right. \\
& \mathbf{S}=\mid \begin{array}{r}
g \cdot c_{l, m x} \cdot A_{m x} \cdot\left(i-J_{m x}\right)-\frac{3}{4} \cdot c_{D} \cdot\left(v_{l, m x}-v_{s, m x}\right)^{2} \cdot \frac{c_{s, m x} \cdot A_{m x}}{d_{50}}+ \\
c_{l, m x} \cdot g \cdot A_{m x} \cdot \frac{n^{2} \cdot\left(V_{c w}-V_{m x}\right)^{2}}{R_{c w}^{4 / 3}}
\end{array} \\
& g \cdot c_{s, m x} \cdot A_{m x} \cdot \frac{\rho_{s}-\rho_{l}}{\rho_{s}} \cdot\left[\left(i^{2}-1\right) \cdot \operatorname{tg} \delta+i\right]+\frac{3}{4} \cdot c_{D} \cdot \frac{\rho_{l}}{\rho_{s}} \cdot\left(v_{l, m x}-v_{s, m x}\right)^{2} . \\
& \frac{c_{s, m x} \cdot A_{m x}}{d_{50}}+c_{s, m x} \cdot g \cdot A_{m x} \cdot \frac{n^{2} \cdot\left(V_{c w}-V_{m x}\right)^{2}}{R_{c w}^{4 / 3}} \\
& \mathbf{C}=\left(\begin{array}{llllll}
0 & 0 & 0 & 1 & c_{l} & c_{s}
\end{array}\right)
\end{aligned}
$$

Numerical treatments of such equations, generally, require schemes capable of preserving discontinuities, possibly without any special shift (shock-capturing schemes). Most numerical approaches have been developed in the last two or three decades, that include the use of finite differences, finite elements or discrete/distinct element methods [1, 19].

The McCormack predictor-corrector explicit scheme is widely used for solving dam-break problems, due to the fact that it is a shock-capturing technique, with second order accuracy both in time and in space, and that the artificial dissipation terms, the so-called Total Variation Diminishing (TVD) Lax-Wendroff correction, can be introduced, in order to avoid non-physical shocks and oscillations around discontinuities $[8,9]$.

The main disadvantage of this solver regards the restriction to the time step size in order to satisfy Courant-Friedrichs-Lewy (CFL) stability condition. However, this is not a real problem for dam-break debris flow phenomena that require short time step to describe the evolution of the discharge. 
Whatsoever the solver adopted, at each timestep the degree of maturity has to be assessed, in order to choose the appropriate terms to incorporate in the SV equations.

\section{Conclusions}

Achieving a set of debris flow constitutive equations is a task which has been given particular attention by the scientific community during the second half of the last century.

In this context, the present paper describes the main features and characteristics of a numerical model suitable to solve the SV equations, modified for including two-phase debris flow phenomena, and able to assess the depth of the wave and the velocities of both the liquid and solid phases of non-stratified (mature) flow, following dam-break events.

The model is based on mass and momentum conservation equations for both liquid and solid phases. The McCormack-Jameson two-step explicit scheme with second order accuracy was employed for the solution of the equations, written in a conservative-law form. The technique was applied for determining both the propagation and the profile of a debris flow wave resulting from the instantaneous and complete collapse of a storage dam. Different experimental cases of dam-break situations in a square section channel were considered for the purpose of comparing results.

Agreements between computational and experimental results regarding both wave front-advance and stage hydrographs are considered very satisfactory.

To widen the reach of the proposed model an essential improvement has been outlined in the paper. This improvement will render the model suitable to predict stratified (immature) flow by taking into account mass and momentum conservation equations for each phase and layer. Momentum conservation equations describe energy exchanges between the two phases in the same layer and between layers, while mass conservation equations describe mass exchanges between layers.

Within this ground, in order to analyse reverse grading (sorting) it is necessary to analyse the wave propagation process, when the solid phase is composed of non-homogeneous material. In this case the model should be further improved in order to feature the distribution of the material of different size of the solid phase: larger size material positioned in the front and in the top of the wave, and finer one in the bottom and in the tail.

\section{References}

[1] Asmar B.N., Lanston P.A., Ergenzinger Z., "The potential of the discrete method to simulate debris flow" in Proceeding of the First International Conference on Debris Flow Hazard Mitigation: Mechanics, Prediction and Assessment, Eds. Chen, New York, 1997 
[2] Aureli F., Mignosa P., Tomirotti M., "Numerical simulation and experimental verification of dam-break flow with shocks" Journal of Hydraulic Research, 2000, 38, pp. 197-216

[3] Bagnold R.A., "Experiments on a gravity-free dispersion of large solid spheres in a Newtonian fluid under shear" in Proceedings of the Royal Society of London, Series A, 225, 1954, pp. 49-63

[4] Bechteler W., Kulisch H., Nujic M., "2D dam-break flooding wave: comparison between experimental and calculated results" Floods and Flood Management, Ed. Saul, Dodrecht, 1992

[5] Bellos V., Sakkas J.G., "1D dam-break flood propagation on dry bed" Journal of Hydraulic Engineering, 1987, ASCE 113(12), pp. 1510 - 1524

[6] Brufau P., Garcia-Navarro P., Ghilardi P., Natale L., Savi F., "1D Mathematical modelling of debris flow" Journal of Hydraulic Research, 38, 2001, pp. $435-446$

[7] Egashira S., Ashida K., "Sediment transport in steep slope flumes", Proc. of RoC Japan Joint Seminar on Water Resources, 1987

[8] Garcia R., Kahawita R.A., "Numerical solution of the De Saint Venant equations with the McCormack finite-difference scheme" International Journal of Numerical Methods in Fluids, 1986, 6, pp. 259-274

[9] Garcia Navarro P., Saviròn J.M., "McCormack methods for numerical simulation of 1D discontinuous unsteady open channel flow" Journal of Hydraulic Research, 1992, 30(1), pp. 313-327

[10] Ghilardi P., Natale L., Savi F., "Two mathematical models simulating a real-world debris-flow", Proc. IAHR Symposium on River, Coastal and Estuarine Morphodynamics, Genova, 1999

[11] Gregoretti C., "Fronte di debris-flow. Composizione e celerità", L'acqua n. 6, pp. 29-39, 1998 (in Italian)

[12] Gregoretti C., "Stima della velocità massima del fronte di una colata detritica che si propaga in un alveo torrentizio", Idra 2000 (in Italian)

[13] Jameson A., "Transonic airfoil calculation using the Euler equations" Numerical Models in Aeronautical Fluid Dynamics, Ed. P.L. Roe, 1982, Academic Press, New York

[14] Larcan E., Mambretti S., Orsi E., "Leggi di resistenza al moto nelle colate di detriti conseguenti al crollo di dighe. 1. Apparato sperimentale e primi risultati." Proceedings on $28^{\circ}$ Convegno di Idraulica e Costruzioni Idrauliche, Potenza, 16-19 settembre 2002 (in Italian)

[15] Larcan E., Mambretti S., Pulecchi M., "A procedure for the evaluation of debris flow stratification." Proceedings of the $1^{\text {st }}$ International Conference on Monitoring, Simulation, Prevention and Remediation of Dense and Debris flow Eds. Lorenzini, Brebbia and Emmauouloudis, 7-9 giugno 2006, Rhodes, Greece

[16] Mambretti S., Larcan E., De Wrachien D., "Debris flow and dam-break surges: Experimental analysis and two-phase modelling" Quaderni di Idronomia Montana, 27, pp. 447-462, 2007 
196 Monitoring, Simulation, Prevention and Remediation of Dense Debris Flows II

[17] Mambretti S., Larcan E., De Wrachien D., "Theoretical and experimental analysis of debris flow: Rehology and two-phase modelling" (in press) Irrigation and Drainage, 2008

[18] McCormack R.W., "The effect of viscosity in hypervelocity impact cratering" AIAA Paper, 1969, 75-1

[19] Rodriguez C., Blanco A., Garcia R., "Comparison of 1D debris flow modelling approaches using a high resolution and non-oscillatory numerical scheme on the finite volume methods", Proceeding of the $1^{\text {st }}$ International Conference on Monitoring, Simulation, Prevention and Remediation of Dense and Debris Flow, Eds. Lorenzini and Brebbia, Rhodes, 2006

[20] Takahashi T. Debris flow International Association for Hydraulic Research, Balkema, Rotterdam, 1991

[21] Wan Z., Wang Y., Hyper-concentrated flow International Association for Hydraulic Research monograph, The Netherlands, 1984 